\title{
Cerebrospinal Fluid Levels of Amyloid Beta 1-43 Mirror 1-42 in Relation to Imaging Biomarkers of Alzheimer's Disease
}

\author{
Ina S. Almdahl1,2*, Camilla Lauridsen ${ }^{3}$, Per Selnes ${ }^{1,2}$, Lisa F. Kalheim ${ }^{1,2}$, \\ Christopher Coello ${ }^{4}$, Beata Gajdzik ${ }^{5}$, Ina Møller ${ }^{6}$, Marianne Wettergreen ${ }^{2,7}$, \\ Ramune Grambaite ${ }^{2}$, Atle Bjørnerud ${ }^{8}$, Geir Bråthen ${ }^{3,6}$, Sigrid B. Sando ${ }^{3,6}$, \\ Linda R. White ${ }^{3,6}$ and Tormod Fladby ${ }^{1,2}$
}

\begin{abstract}
' Division of Medicine and Laboratory Sciences, Institute of Clinical Medicine, Faculty of Medicine, University of Oslo, Oslo, Norway, ${ }^{2}$ Department of Neurology, Akershus University Hospital, Lørenskog, Norway, ${ }^{3}$ Department of Neuroscience, Faculty of Medicine, Norwegian University of Science and Technology, Trondheim, Norway, ${ }^{4}$ Preclinical PET/CT, Institute of Basic Medical Sciences, University of Oslo, Oslo, Norway, ${ }^{5}$ Aleris, Oslo, Norway, ${ }^{6}$ Department of Neurology and Clinical Neurophysiology, University Hospital of Trondheim, Trondheim, Norway, ${ }^{7}$ Department of Clinical Molecular Biology (EpiGen), Institute of Clinical Medicine, University of Oslo - Akershus University Hospital, Lørenskog, Norway, ${ }^{8}$ The Intervention Centre, Oslo University Hospital, Oslo, Norway
\end{abstract}

\section{OPEN ACCESS}

Edited by:

Catarina Oliveira,

University of Coimbra, Portugal

Reviewed by:

Ramesh Kandimalla,

Texas Tech University, USA

Panteleimon Giannakopoulos, University of Geneva, Switzerland Ines Baldeiras,

University of Coimbra, Portugal

*Correspondence: Ina S. Almdahl

ina.almdahl@medisin.uio.no

Received: 17 October 2016 Accepted: 12 January 2017

Published: 07 February 2017

Citation:

Almdahl IS, Lauridsen $C$, Selnes $P$, Kalheim LF, Coello C, Gajdzik B,

Møller I, Wettergreen $M$,

Grambaite R, Bjørnerud A,

Bråthen $G$, Sando $S B$, White $L R$ and Fladby T (2017) Cerebrospinal Fluid Levels of Amyloid Beta 1-43 Mirror 1-42 in Relation to Imaging Biomarkers of Alzheimer's Disease.

Front. Aging Neurosci. 9:9. doi: 10.3389/fnagi.2017.00009
Introduction: Amyloid beta 1-43 (A $\beta 43)$, with its additional C-terminal threonine residue, is hypothesized to play a role in early Alzheimer's disease pathology possibly different from that of amyloid beta 1-42 (A $\beta 42$ ). Cerebrospinal fluid (CSF) A $\beta 43$ has been suggested as a potential novel biomarker for predicting conversion from mild cognitive impairment $(\mathrm{MCl})$ to dementia in Alzheimer's disease. However, the relationship between CSF A 433 and established imaging biomarkers of Alzheimer's disease has never been assessed.

Materials and Methods: In this observational study, CSF A $\beta 43$ was measured with ELISA in 89 subjects; 34 with subjective cognitive decline (SCD), 51 with $\mathrm{MCl}$, and four with resolution of previous cognitive complaints. All subjects underwent structural MRI; 40 subjects on a $3 \mathrm{~T}$ and 50 on a 1.5T scanner. Forty subjects, including 24 with SCD and 12 with $\mathrm{MCl}$, underwent ${ }^{18} \mathrm{~F}$-Flutemetamol PET. Seventy-eight subjects were assessed with ${ }^{18} \mathrm{~F}$-fluorodeoxyglucose PET (21 SCD/7 MCl and $11 \mathrm{SCD} / 39 \mathrm{MCl}$ on two different scanners). Ten subjects with SCD and 39 with $\mathrm{MCl}$ also underwent diffusion tensor imaging.

Results: Cerebrospinal fluid $A \beta 43$ was both alone and together with $p$-tau a significant predictor of the distinction between SCD and $\mathrm{MCl}$. There was a marked difference in CSF A 343 between subjects with ${ }^{18} \mathrm{~F}$-Flutemetamol PET scans visually interpreted as negative (37 pg/ml, $n=27$ ) and positive ( $15 \mathrm{pg} / \mathrm{ml}, n=9), p<0.001$. Both CSF $A \beta 43$ and $A \beta 42$ were negatively correlated with standardized uptake value ratios for all analyzed regions; CSF $A \beta 43$ average rho -0.73, $A \beta 42-0.74$. Both CSF $A \beta$ peptides correlated significantly with hippocampal volume, inferior parietal and frontal cortical thickness and axial diffusivity in the corticospinal tract. There was a trend toward CSF A $\beta 42$ being better correlated with cortical glucose metabolism. None of the studied correlations between CSF $A \beta 43 / 42$ and imaging biomarkers were significantly different for the two $A \beta$ peptides when controlling for multiple testing. 
Conclusion: Cerebrospinal fluid A $\beta 43$ appears to be strongly correlated with cerebral amyloid deposits in the same way as $A \beta 42$, even in non-demented patients with only subjective cognitive complaints. Regarding imaging biomarkers, there is no evidence from the present study that CSF A 343 performs better than the classical CSF biomarker A $\beta 42$ for distinguishing SCD and $\mathrm{MCl}$.

Keywords: Alzheimer's disease, amyloid beta 1-43, cerebrospinal fluid, positron emission tomography, magnetic resonance imaging, mild cognitive impairment

\section{INTRODUCTION}

Alzheimer's disease (AD) is the leading cause of dementia. Treatment of this devastating disease will depend on biomarkers that can reliably identify individuals who will develop dementia due to $\mathrm{AD}$ in the future. A previous study following patients with mild cognitive impairment (MCI) for 2 years, found that the baseline cerebrospinal fluid (CSF) levels of amyloid-beta 1-43 $(\mathrm{A} \beta 43)$ could distinguish patients that converted to $\mathrm{AD}$ dementia from those that did not, suggesting that CSF A $\beta 43$ could be a useful addition to the more well-studied CSF biomarkers amyloid beta 1-42 (A $\beta 42)$, total tau ( $\mathrm{t}$-tau), and tau phosphorylated on position 181 (p-tau) (Kandimalla et al., 2011, 2013; Lauridsen et al., 2016). A $\beta 43$ differs from $A \beta 42$ by one $C$-terminal threonine residue, and is the product of an alternative $\gamma$-secretase cleavage pathway from the amyloid precursor protein (APP) (Takami et al., 2009). Findings from studies of neuropathology, genetics and animal models have resulted in the hypothesis that $\mathrm{A} \beta 43$ could play a role in $\mathrm{AD}$ pathogenesis out of proportion to its low levels in the brain. With its additional C-terminal betabranched amino acid, A $\beta 43$ could theoretically be expected to be more prone to aggregation than $A \beta 42$. Experiments in vitro have yielded conflicting results: some report that $A \beta 43$ indeed aggregates faster than $\mathrm{A} \beta 42$ and with a higher potential for seeding aggregation of other $A \beta$ species (Saito et al., 2011; Conicella and Fawzi, 2014), others that A $\beta 43$ aggregates slower with later amyloid nucleation and that it is inefficient in crossseeding $A \beta 42$ (Chemuru et al., 2016). Whether these experiments reflect the true aggregational process in the human brain is uncertain (Vandersteen et al., 2012). Cerebral deposition of $A \beta 43$ is frequently present both in sporadic and familial AD (Welander et al., 2009; Keller et al., 2010; Sandebring et al., 2013) as a component of both neuritic and diffuse extracellular plaques (Iizuka et al., 1995; Parvathy et al., 2001; Miravalle et al., 2005). Some PSEN1 mutations associated with familial AD are known to cause an overproduction of A $\beta 43$ (Nakaya et al., 2005; Shimojo et al., 2008). In a transgenic mouse model based on such a PSEN1 mutation, A $\beta 43$ appeared to have greater neurotoxicity than $A \beta 42$ with short-term memory impairment occurring with rising levels of $\mathrm{A} \beta 43$ even before plaque formation (Saito et al., 2011). A $\beta 43$ has also been shown to deposit ahead of $A \beta 42$ in the brain of mutant APP transgenic mice (Zou et al., 2013).

Information is sparse regarding CSF A $\beta 43$ as a potential clinical biomarker, especially in early stages of cognitive impairment. Previously, it has been shown that CSF A $\beta 43$ levels are decreased in $\mathrm{MCI}$ and $\mathrm{AD}$ dementia as compared to controls, with a strong correlation between CSF levels of $\mathrm{A} \beta 43$ and $\mathrm{A} \beta 42$ (Kakuda et al., 2012; Lauridsen et al., 2016). At the late stage of dementia, CSF $A \beta 43$ and $A \beta 42$ appear to have equal diagnostic accuracy for discriminating AD dementia from non-demented controls (Bruggink et al., 2013). Clinically more important, however, is the ability of biomarkers to single out non-demented patients that are on a trajectory toward AD dementia. A meta-analysis combining the classical CSF biomarkers; A $\beta 42$ with t-tau and/or p-tau, yielded a mean sensitivity of $84 \%$ and a mean specificity of $63 \%$ for the distinction between stable and progressive MCI (Ferreira et al., 2014). Enhancement of this diagnostic performance would obviously be an advantage. Lauridsen et al. (2016) found that when used in a ratio with t-tau, substituting $A \beta 42$ with $A \beta 43$ gave a slight, but significant improvement of the diagnostic accuracy for this distinction, a finding that warrants further exploration. The use of CSF biomarkers in clinical routine is impeded by the invasiveness of lumbar puncture and by the high between-center variability particularly in the measurement of $\mathrm{A} \beta 42$. Imaging biomarkers are often more readily available, provide complimentary information as well as improve the predictive accuracy for dementia conversion when combined with CSF biomarkers (Vemuri et al., 2009). To our knowledge, CSF $A \beta 43$ has not been described in relation to imaging biomarkers.

Positron emission tomography (PET) imaging allows visualization of cerebral $A \beta$ aggregates in vivo. Uptake of amyloid-binding PET tracers, like ${ }^{18} \mathrm{~F}$-Flutemetamol $\left({ }^{18} \mathrm{~F}\right.$ FLUT), correlates inversely with CSF A $\beta 42$ levels (Fagan et al., 2006; Li et al., 2015), and positively with $\mathrm{A} \beta$ plaque burden observed post-mortem (Ikonomovic et al., 2008). It remains to be determined whether the relationship with amyloid PET is the same for CSF $A \beta 43$. In addition to amyloid pathology, development of $\mathrm{AD}$ is characterized by neurodegeneration. Neurodegenerative changes in AD identifiable by magnetic resonance imaging (MRI) include gray matter atrophy of the hippocampus and vulnerable cortical regions (Whitwell et al., 2008; Sabuncu et al., 2011), and microstructural white matter changes resulting in increased mean, radial and axial diffusivity and reduced fractional anisotropy on diffusion tensor imaging (DTI) (Selnes et al., 2013; Amlien and Fjell, 2014; Lee et al., 2015). Several studies have reported correlations between CSF $A \beta 42$ and structural MRI, while others have found no association, with methodological differences suggested as a possible reason for the discrepancy (Vemuri and Jack, 2010; Li et al., 2014). Neurodegeneration is also related to changes in cerebral metabolism as assessed by ${ }^{18} \mathrm{~F}$-fluorodeoxyglucose $\left({ }^{18}\right.$ F-FDG) PET imaging (Fouquet et al., 2009). In AD dementia 
cortical ${ }^{18} \mathrm{~F}-\mathrm{FDG}$ uptake has been reported to correlate with CSF A $\beta 42$ levels (Vukovich et al., 2009; Yakushev et al., 2012) and ${ }^{18}$ F-FDG PET imaging appears to have high prognostic value in MCI (Shaffer et al., 2013; Perani et al., 2016).

The objectives of this study were to explore firstly whether CSF A $\beta 43$ reflects cerebral amyloid deposits as visualized by ${ }^{18}$ F-FLUT PET, and secondly whether CSF A $\beta 43$ correlates with MRI and ${ }^{18} \mathrm{~F}$-FDG PET imaging findings of neurodegeneration in non-demented patients with cognitive complaints.

\section{MATERIALS AND METHODS}

\section{Subject Recruitment Cohort 1 - Amyloid PET cohort:}

Forty subjects were included in the Dementia Disease Initiation (DDI) project at Akershus University Hospital between March 2013 and March 2016. They were referred by their general practitioner to the hospital's memory clinic or recruited through newspaper advertisements. Inclusion criteria were complaints of decline in cognitive capacity compared with a previously normal state, age 40-79 and Scandinavian first language. Exclusion criteria were established dementia, neurodevelopmental disorders, known brain injury including recognized previous stroke, as well as any serious somatic or psychiatric disorder or drug use that could significantly influence cognitive capacity. All subjects were assessed with ${ }^{18}$ F-FLUT PET either at the time of inclusion $(n=22)$ or at a second assessment 2 years after first inclusion in the project $(n=18)$. Clinical assessment, lumbar puncture, and MRI were done within 3.5 months of ${ }^{18}$ F-FLUT PET. Thirtyone of the 40 subjects also underwent ${ }^{18}$ F-FDG PET. Patients were interviewed and examined by a physician trained in diagnosing cognitive disorders. A clinical report form was used to collect information about current cognitive symptoms both from the participant and a knowledgeable informant. Standardized cognitive testing, physical examination, and blood screening were completed. MCI $(n=12)$ was defined based on the core criteria in the recommendation from the National Institute on Aging-Alzheimer's Association (NIA/AA; Albert et al., 2011). Documented impairment greater than expected for the person's age, gender, and educational level in one or more cognitive domains was operationalized by a score 1.5 standard deviations or more below the normative mean on at least one of the following tests; the delayed recall task of the CERAD Word List Test (Fillenbaum et al., 2008), Trail Making Test B (TMTB) (Reitan and Wolfson, 1985), Controlled Oral Word Association Test (COWAT) (Benton and Hamsher, 1989) and the silhouettes task from the Visual Object and Space Perception (VOSP) Battery (Warrington and James, 1991) or a score below 28 on MMSE (Folstein et al., 1975). All subjects maintained independent functioning in social, and if appropriate, occupational settings, and had a global Clinical Dementia Rating score of $\leq 0.5$ (Morris, 1997). Subjective cognitive decline (SCD) ( $n=24$ ) was defined according to the recommendations by the Subjective Cognitive Decline Initiative Working Group (Jessen et al., 2014), with normal performance on standardized cognitive tests operationalized by a score above 1.5 standard deviations below the normative mean on the above mentioned tests. Four subjects had been classified as having SCD at inclusion, but did not have cognitive complaints 2 years later when they underwent ${ }^{18}$ F-FLUT PET. They had normal performance on cognitive tests and were classified as cognitively normal with resolution of previous cognitive complaints $(\mathrm{CN})$.

\section{Cohort 2 - MRI and DTI cohort:}

Fifty subjects were included in the MCI project at Akershus University Hospital between January 2007 and February 2013 after having been referred to the hospital's memory clinic by their general practitioner. Inclusion criteria were cognitive complaints for at least 6 months and age 40-79. Exclusion criteria included established dementia, major psychiatric disorder, drug abuse, significant solvent exposure, and anoxic brain damage. All subjects underwent lumbar puncture and MRI at inclusion. The subjects were assessed with clinical interview, routine physical examination, blood screening, and a battery of cognitive tests. One subject was found to have been included in Cohort 1 and was therefore excluded from Cohort 2 when data from both cohorts were analyzed together (total number of unique subjects in the study $n=89$ ). Subjects in Cohort 2 were defined as having MCI $(n=39)$ if objective cognitive impairment was evident on at least one of the following screening tests; MMSE score below 28, score equivalent to mild impairment on one or more of the items of the Cognistat (Kiernan et al., 1987) or score $>1$ on I-Flex (Royall et al., 1992). Subjects without objective cognitive impairment on the same screening battery were classified as having SCD $(n=11)$.

\section{Ethics Statement}

The study was conducted in accordance with the Helsinki Declaration. All participants gave written informed consent. The Regional Committee for Medical and Health Research Ethics, South East Norway, approved the study (approval 2009/2550 and 2013/150).

\section{CSF Collection and Storage}

Lumbar puncture was performed generally between 8 a.m. and noon, at the L3/L4, L4/L5, or L5/S1 interspace and without any serious adverse events. The first $4 \mathrm{ml}$ CSF was used for routine clinical investigations. The next 1.5 and $4.5 \mathrm{ml} \mathrm{CSF}$ were collected in two polypropylene tubes and centrifuged at $2000 \times g$ for $10 \mathrm{~min}$ within $4 \mathrm{~h}$ of collection. The $1.5 \mathrm{ml} \mathrm{CSF}$ was stored at $-80^{\circ} \mathrm{C}$ prior to analysis of the traditional CSF biomarkers $\mathrm{A} \beta 42$, t-tau and p-tau. In Cohort 1, the $4.5 \mathrm{ml} \mathrm{CSF}$ was aliquoted into $450 \mu \mathrm{l}$ polypropylene tubes before storage at $-80^{\circ} \mathrm{C}$, while in Cohort 2 the $4.5 \mathrm{ml} \mathrm{CSF}$ was stored at $-80^{\circ} \mathrm{C}$, before later being thawed and aliquoted, with further storage at $-80^{\circ} \mathrm{C}$ prior to determination of $\mathrm{A} \beta 43$. Consequently, the samples underwent one freeze-thaw cycle before determination of $A \beta 43$ in Cohort 1 and two in Cohort 2, with the exception of two samples in Cohort 2 where due to lack of CSF in the biobank, remaining CSF after analysis of the traditional CSF biomarkers was used also for analysis of $\mathrm{A} \beta 43$, resulting in three freeze-thaw cycles. 


\section{ELISA Assays and APOE Genotyping}

Cerebrospinal fluid levels of $A \beta 42$, $t$-tau, and $p$-tau were quantified with commercially available ELISAs; Innotest ${ }^{\circledR}$ $\beta$-amyloid 1-42 (Vanderstichele et al., 2000), Innotest ${ }^{\oplus}$ hTau Ag (Blennow et al., 1995), and Innotest ${ }^{\circledR}$ phosphoTau (181P) (Vanmechelen et al., 2000) (Fujirebio Europe, Gent, Belgium), and carried out in accordance with the manufacturers' instructions at the national reference laboratory for these tests at the Department of Interdisciplinary Laboratory Medicine and Medical Biochemistry, Akershus University Hospital. The laboratory lists the following cut-off values for abnormality (modified from Sjögren et al., 2001); t-tau $>300 \mathrm{pg} / \mathrm{ml}$ for age $<50$ years, $>450 \mathrm{pg} / \mathrm{ml}$ for age 50-69 years, and $>500 \mathrm{pg} / \mathrm{ml}$ for age $\geq 70$ years, $\mathrm{p}$-tau $\geq 80 \mathrm{pg} / \mathrm{ml}$ and A $\beta 42<550 \mathrm{pg} / \mathrm{ml}$.

$\mathrm{A} \beta 43$ in CSF was analyzed at the laboratory of the Department of Neuroscience, Norwegian University of Science and Technology, Trondheim, Norway, with an ELISA monoplex kit; A $\beta 1-43$, RE59711 (IBL, Hamburg, Germany) run according to the instructions given by the manufacturer. The antibodies included in the kit were anti-human $A \beta$ (38-43) rabbit IgG affinity purity and anti-human $\mathrm{A} \beta$ (82E1) mouse IgG MoAb Fab' affinity purity. According to the manufacturers of the kits the cross-reactivity for $A \beta 42$ in the A $\beta 43$ ELISA is $<1 \%$ and the antibodies in the Innotest ${ }^{\circledR} \beta$-amyloid 1-42 have 50x less affinity for $A \beta 43$ compared to $A \beta 42$. Samples of CSF were analyzed undiluted and in duplicate. The measurement range for the kit was reported to be $2.34-150 \mathrm{pg} / \mathrm{ml}$. All samples analyzed in the study $(7.51-66.49 \mathrm{pg} / \mathrm{ml})$ fell within this range. Intra- and interassay variations have been reported previously (Lauridsen et al., 2016). As this study continued from the previously published material, these values were not calculated again.

Apolipoprotein E (APOE) genotyping was performed on EDTA blood samples from all subjects at the Gene Technology Division, Department of Interdisciplinary Laboratory Medicine and Medical Biochemistry, Akershus University Hospital according to the laboratory's routine protocol using real-time PCR combined with a TaqMan assay (Applied Biosystems, Thermo Fisher Scientific, Waltham, MA, USA).

\section{MRI Imaging Acquisition and Processing}

In Cohort 1, MRI scans were acquired on a Philips Achieva 3 Tesla system. A single 3D turbo field echo sequence was acquired for morphometric analysis with the following sequence parameters: $\mathrm{TR} / \mathrm{TE} / \mathrm{TI} / \mathrm{FA}=4.5 \mathrm{~ms} / 2.2 \mathrm{~ms} / 853 \mathrm{~ms} / 8^{\circ}$, matrix $=256 \times 213,170$ slices, thickness $=1.2 \mathrm{~mm}$, in-plane resolution of $1 \mathrm{~mm} \times 1.2 \mathrm{~mm}$. In Cohort 2, MRI was performed on a Siemens Espree $1.5 \mathrm{~T}$ scanner. One 3D magnetization-prepared rapid gradient echo T1-weighted sequence was obtained with the following specifications: $\mathrm{TR} / \mathrm{TE} / \mathrm{TI} / \mathrm{FA}=2400 \mathrm{~ms} / 3.65 \mathrm{~ms} / 1000 \mathrm{~ms} / 8^{\circ}$, matrix $=240 \times 192,160$ slices, thickness $=1.2 \mathrm{~mm}$, in-plane resolution of $1 \mathrm{~mm} \times 1.2 \mathrm{~mm}$. The pulse sequence used for DTI was: $b=750,12$ directions repeated five times, five b0-values per slice, $\mathrm{TR}=6100 \mathrm{~ms}$, $\mathrm{TE}=117 \mathrm{~ms}$, number of slices $=30$, slice thickness $=3 \mathrm{~mm}(\mathrm{gap}=1.9 \mathrm{~mm})$, in-plane resolution $=1.2 \times 1.2 \mathrm{~mm}^{2}$, bandwidth $=840 \mathrm{~Hz} /$ pixel. Cortical reconstruction and volumetric segmentation was performed with the FreeSurfer image analysis suite version 5.3.01. This includes segmentation of the subcortical white matter and deep gray matter volumetric structures (Fischl et al., 2002) and parcellation of the cortical surface (Fischl et al., 2004) according to a previously published scheme labeling cortical sulci and gyri (Desikan et al., 2006), and thickness values are calculated over the cortical mantle. The thickness value of the entorhinal cortex (ERC) was calculated using a method based on ultra-high resolution ex vivo applied to in vivo MRI, as implemented in FreeSurfer (Fischl et al., 2009). In addition to hippocampal volume and cortical thickness of the ERC, the thickness of the following cortical regions of interest (ROIs) known to be atrophic relatively early in the development of $\mathrm{AD}$ were selected for analysis; the temporopolar, middle temporal, posterior cingulate, inferior parietal, and inferior frontal cortex. For analyses of the total hippocampal volume, the sum of the right and left hippocampal volumes as permillage $(\%)$ of the estimated total intracranial volume was used. For each cortical ROI, the average of the measurements from the right and left hemisphere was used. Image processing for DTI has been described previously (Kalheim et al., 2016). DTI data were missing for one subject $(n=49)$. The fractional anisotropy, mean, radial, and axial diffusivities were assessed in the following four tracts, selected based on previous reports of DTI changes in AD and MCI (Amlien and Fjell, 2014; Lee et al., 2015) and calculated as an average of the metrics from the right and left hemispheres; the cingulum bundles (average of the cingulumcingulate gyrus bundle and the cingulum-angular bundle), the corpus callosum-forceps bundles (average of the corpus callosum bundles to forceps major and minor, respectively), the uncinate fasciculus and the corticospinal tract.

\section{PET Imaging Acquisition, Processing, and Interpretation}

In Cohort $1{ }^{18} \mathrm{~F}$-FLUT and ${ }^{18} \mathrm{~F}$-FDG PET/CT imaging were performed on the same GE Discovery $690 \mathrm{PET} / \mathrm{CT}$ scanner on two separate days. Subjects received a bolus injection of $185 \mathrm{MBq}(5 \mathrm{mCi})$ tracer and after resting were positioned headfirst supine in the scanner. A low-dose CT scan was acquired first for attenuation correction. Subjects fasted at least $6 \mathrm{~h}$ in advance and blood glucose was measured routinely before ${ }^{18} \mathrm{~F}-$ FDG injection (all subjects had blood glucose below $8.0 \mathrm{mmol} / \mathrm{l}$ ). PET scanning in 3D-mode commenced $45 \mathrm{~min}$ after injection of ${ }^{18} \mathrm{~F}$-FDG and $90 \mathrm{~min}$ after ${ }^{18} \mathrm{~F}$-FLUT. PET data were acquired for $10 \mathrm{~min}$ for ${ }^{18} \mathrm{~F}$-FDG and for $20 \mathrm{~min}$ (four frames of $5 \mathrm{~min}$ ) for ${ }^{18} \mathrm{~F}$-FLUT. Acquired data were corrected for random events, dead time, attenuation, scatter, and decay. PET volumes were reconstructed with an iterative algorithm (VUE Point FX SharpIR with six iterations, 24 subsets for ${ }^{18} \mathrm{~F}$-FDG, four iterations, 16 subsets for ${ }^{18} \mathrm{~F}$-FLUT) and smoothed with a post-reconstruction 3D Gaussian filter of $3 \mathrm{~mm}$ full-width at half maximum. Image format for ${ }^{18} \mathrm{~F}-\mathrm{FDG}$ was $256 \times 256$ (pixel size $1 \mathrm{~mm} \times 1 \mathrm{~mm}$ ), for ${ }^{18}$ F-FLUT $192 \times 192$ (pixel size $1.3 \mathrm{~mm} \times 1.3 \mathrm{~mm}$ ), with slice

${ }^{1}$ http://surfer.nmr.mgh.harvard.edu/ 
thickness $3.75 \mathrm{~mm}$. In Cohort $2{ }^{18} \mathrm{~F}$-FDG PET/CT-scans were acquired as previously described (Coello et al., 2013).

Visual interpretation of the ${ }^{18}$ F-FLUT images was done by trained readers and the scans were classified as positive or negative in line with the manufacturer's guidelines. For the automated quantitative assessment, motion correction of the dynamic ${ }^{18}$ F-FLUT PET was performed using frame by frame rigid registration, then the frames were summed to a single time-frame image and registered to the anatomical MRI volume using a six-parameter rigid registration as implemented in the Spatial Parametrical Mapping (SPM 12, Wellcome Trust Centre for Neuroimaging, UCL, UK) toolbox. Due to missing dynamic images one subject had to be excluded from the automated quantitative analyses $(n=39)$. Five cortical ROIs known to harbor substantial amyloid plaques in $\mathrm{AD}$ were selected for analysis of ${ }^{18} \mathrm{~F}$-FLUT uptake: the precuneus and posterior cingulate combined, anterior cingulate, prefrontal, inferior parietal and lateral temporal cortex. The average ${ }^{18}$ F-FLUT uptake in each of these ROIs was calculated incorporating values from both hemispheres. The average uptake in the cerebellar cortex, which is usually devoid of amyloid pathology in early $\mathrm{AD}$, was used as the reference region after eroding voxels at the segmentation boundaries to avoid influence due to inaccurate segmentation or co-registration. Regional standardized uptake value ratios (SUVRs) for ${ }^{18}$ F-FLUT were created by dividing the average uptake in each ROI by the average uptake in the cerebellar cortex.

The same ROIs that were used for the structural MRI analyses were selected for study of ${ }^{18}$ F-FDG activity. Uptake in the cerebellar white matter was used as the reference region after first eroding the cerebellar white matter mask. SUVRs were calculated by dividing the average uptake of ${ }^{18} \mathrm{~F}$-FDG per voxel in each ROI to the average uptake in the cerebellar white matter.

\section{Statistical Analysis}

The statistical analyses were performed using IBM SPSS version 23 (Chicago, IL, USA) unless otherwise stated. All tests were two-sided and $p$-values below 0.05 were considered significant. Distribution of the variables and whether normal distribution could be assumed were assessed by histograms and a ShapiroWilk test. Levene's statistics were calculated to assess the homogeneity of variance in each variable for parametric tests. For comparisons of CSF biomarker levels, demographical data and neuropsychological test results between SCD and MCI, a $\chi^{2}$ test was used for categorical variables, an independent sample $t$-test for continuous variables with normal distribution, and a Mann-Whitney $U$ test for continuous variables with non-normal distribution. Binary logistic regression models were created with the SCD/MCI distinction as the dependent variable and with either CSF tau, p-tau or one of the imaging biomarkers as a covariate. In significant models, CSF $A \beta 43$ and $A \beta 42$ were then in turn added as a second covariate. Bivariate correlation and partial correlation controlling for age were assessed between CSF biomarkers, MRI, ${ }^{18}$ F-FLUT and ${ }^{18}$ F-FDG variables. Spearman's rank coefficients ( $r h o$ ) of the correlations between an imaging variable and $A \beta 43$, and the imaging variable and $A \beta 42$, were compared with an asymptotic $z$ test using software available from http://quantpsy.org (Lee and Preacher, 2013). To detect potential interrelating effects of aging, all analyses were done both unadjusted and with age as a covariate. Controlling for gender and educational length was not done, as these factors were not found to have significant impact in linear regression models of imaging measures as a function of $\mathrm{A} \beta$. APOE genotype was related to CSF $A \beta 43$ levels, but was not found to be a significant factor in regression models that already included either $A \beta 42$ or $A \beta 43$, and was therefore not included as a covariate. Differences in baseline CSF A $\beta 43$ and $A \beta 42$ levels between groups based on the result of the ${ }^{18}$ F-FLUT PET were assessed using an independent samples $t$-test. Receiver operating characteristic (ROC) curves for the prediction of a positive ${ }^{18} \mathrm{~F}$ FLUT scan were plotted for both $\mathrm{A} \beta$ peptides, and area under the curve (AUC) was calculated. Cut-off values for $A \beta 43$ and $A \beta 42$ yielding the best combination of sensitivity and specificity, were determined by maximal Youden's index. Differences in AUC were assessed using MedCalc statistical software (MedCalc software, Mariakerke, Belgium).

\section{RESULTS}

\section{Demographics, Cognition, and CSF Biomarkers: Comparison between SCD and $\mathrm{MCl}$}

Demographical characteristics, cognitive scores and CSF biomarker levels in the SCD and MCI groups are reported in Table 1. The frequency of the APOE\&4 genotype was similar in both SCD and MCI. The pattern of relative levels of the two CSF A $\beta$ peptides was similar with respect to APOE genotype, with significantly lower mean peptide levels in the group with $A P O E \varepsilon 4 / \varepsilon 4$ (Figure 1). No significant difference for the correlations between $A P O E$ allele and $A \beta 43$ and $A \beta 42$ levels was found. There was a strong positive correlation between the CSF measurements of $\mathrm{A} \beta 43$ and $\mathrm{A} \beta 42$ (all subjects rho $0.88, p<0.001)$ with no significant difference between the groups (SCD rho 0.81 and MCI rho 0.86). In the MCI group both $A \beta 43$ and $A \beta 42$ correlated inversely with $t$-tau and $p$-tau without any significant difference between the two amyloid peptides (MCI $n=51$, A $\beta 43: t-$ tau rho $-0.45, p=0.001$, A $\beta 42: t-$ tau rho $-0.35, p=0.01$, A $\beta 43$ :p-tau rho $-0.38, p=0.007$, $\mathrm{A} \beta 42$ :p-tau rho $-0.40, p=0.003)$. In the SCD group, however, there were no significant correlations between $\mathrm{A} \beta 43 / \mathrm{A} \beta 42$ and t-tau and p-tau. For all subjects $(n=89)$ the overall correlation coefficients for $\mathrm{A} \beta 43$ :t-tau was rho -0.28 , $\mathrm{A} \beta 42$ :t-tau rho -0.25 , $\mathrm{A} \beta 43$ :p-tau rho -0.29 , and for $\mathrm{A} \beta 42$ :p-tau rho -0.37 , without any significant differences between $A \beta 43$ and A $\beta 42$. Adjustment for age did not significantly change the correlations between the CSF biomarkers. In binary logistic regression models for the distinction between $\mathrm{SCD}$ and $\mathrm{MCI}$, both $\mathrm{A} \beta 43, \mathrm{~A} \beta 42$, and $\mathrm{p}$-tau were statistically significant predictors when entered into the model as the only CSF 
TABLE 1 | Demographics, cognitive scores, and cerebrospinal fluid (CSF) biomarkers in SCD and MCI.

\begin{tabular}{|c|c|c|c|c|c|}
\hline \multirow[b]{2}{*}{$n$} & \multicolumn{2}{|c|}{ SCD } & \multicolumn{2}{|c|}{$\mathrm{MCl}$} & \multirow[t]{2}{*}{$p$} \\
\hline & 34 & & 51 & & \\
\hline Gender m/f, $n$ & $15 / 19$ & & 22/29 & & - \\
\hline Age & 64.5 & [9] & 65 & {$[10]$} & - \\
\hline Years of education & 14 & {$[4]$} & 14 & [5] & - \\
\hline APOE $\varepsilon 4(\%)$ & 47 & & 45 & & - \\
\hline MMSE total score & 29 & [1] & 28 & [2] & $<0.001$ \\
\hline RAVLT delayed recall $t$-score & 57 & [20] & 47 & {$[18]$} & $<0.001$ \\
\hline TMT B $t$-score & 49 & [9] & 44 & {$[11]$} & 0.005 \\
\hline COWAT $t$-score & 52 & [13] & 49 & [16] & - \\
\hline CSF A 343 pg/ml & 37 & [22] & 24 & [19] & 0.004 \\
\hline CSF A $\beta 42$ pg/ml (\% below cut-off) & 981 & [488] (12) & 679 & [388] (26) & $0.009(0.17)$ \\
\hline CSF t-tau pg/ml (\% above cut-off) & 312 & [155] (6) & 335 & [267] (31) & $0.13(0.006)$ \\
\hline CSF p-tau pg/ml (\% above cut-off) & 57 & [25] (6) & 69 & [34] (35) & $0.001(0.003)$ \\
\hline
\end{tabular}

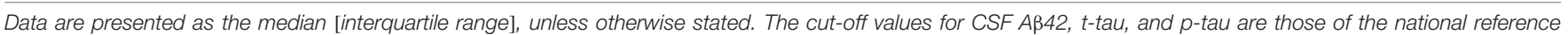

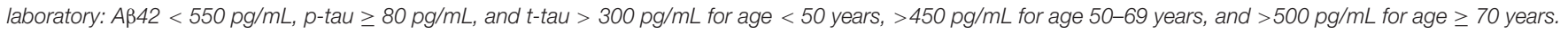

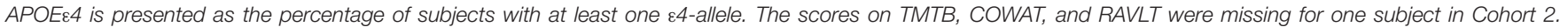

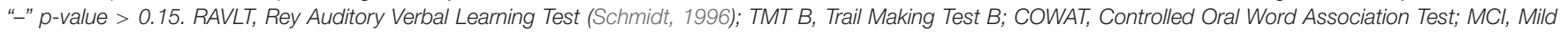
cognitive impairment; SCD, subjective cognitive decline; APOE, Apolipoprotein E genotype.

biomarker. In a multivariate model with p-tau, inclusion of $\mathrm{A} \beta 43$ added significantly to the prediction, while $\mathrm{A} \beta 42$ did not (Table 2).

\section{Amyloid PET}

Based on visual interpretation nine of the $40{ }^{18} \mathrm{~F}$-FLUT PET scans were deemed to be positive (five SCD, four MCI), two borderline positive (one $\mathrm{CN}$ and one $\mathrm{MCI}$ ), two borderline negative (one SCD and one MCI), and 27 negative (18 SCD, six MCI, three $\mathrm{CN})$. The mean CSF $\mathrm{A} \beta 43$ in these four groups were 15,22 , 32, and $37 \mathrm{pg} / \mathrm{ml}$, respectively, and the mean CSF A $\beta 42$ 547, 657, 1074, and $1042 \mathrm{pg} / \mathrm{ml}$. The mean difference [95\% CI] in CSF concentration between subjects with positive and negative scans was $22 \mathrm{pg} / \mathrm{ml}[13,31]$ for $\mathrm{A} \beta 43$ and $495 \mathrm{pg} / \mathrm{ml}[380,611]$ for $\mathrm{A} \beta 42, p<0.001$ for both. ROC curves for prediction of a positive scan gave AUC 0.97 for both $\mathrm{A} \beta 43$ and $\mathrm{A} \beta 42$. The best cut-off value was $\leq 24 \mathrm{pg} / \mathrm{ml}$ for $\mathrm{A} \beta 43$ with sensitivity $100 \%$ and specificity $93 \%$ for a positive scan, and $\leq 679 \mathrm{pg} / \mathrm{ml}$ for $\mathrm{A} \beta 42$ with sensitivity $100 \%$ and specificity $89 \%$. In the group with negative scans, 16/27 (59\%) had APOE genotype APOE\&3/\&3 and 9/27

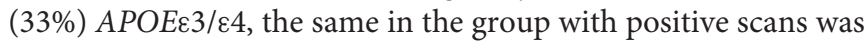
$2 / 9(22 \%)$ and $5 / 9(56 \%)$, but the differences were not statistically significant. ${ }^{18}$ F-FLUT SUVR based on the automated quantitative assessment of the scans was not a significant predictor of the SCD/MCI distinction in binary logistic regression models with and without age as a covariate. Mean overall ${ }^{18} \mathrm{~F}$-FLUT SUVR for the five ROIs was 1.29, 95\% CI [1.17-1.40] in the SCD group and 1.40, 95\% CI [1.23-1.58] in the MCI group $(p=0.26)$. There were highly significant inverse correlations between CSF A $\beta 43$ and ${ }^{18}$ F-FLUT SUVR in all the examined ROIs and the correlations became stronger with adjustment for the effect of age (Table 3). The same was true for $\mathrm{A} \beta 42$, and there were no significant differences between the correlation coefficients for the two $A \beta$ peptides. The correlations with overall ${ }^{18}$ F-FLUT SUVR remained strong also when analyzing only subjects diagnosed with SCD $(n=23$; rho $-0.64, p=0.001$ for both $\mathrm{A} \beta$ peptides, adjusted for age rho $-0.69, p<0.001$ for $\mathrm{A} \beta 43$, and rho $-0.67, p=0.001$ for $\mathrm{A} \beta 42$ ). When excluding subjects with visually interpreted definitely positive scans from the analysis $(n=31)$, there were still significant correlations with overall SUVR; for A $\beta 43$ unadjusted rho $-0.37, p=0.04$, adjusted for age rho $-0.52, p=0.004$, for $\mathrm{A} \beta 42$ unadjusted rho $-0.39, p=0.03$, adjusted for age rho $-0.51, p=0.004$. CSF t-tau and p-tau were not significantly correlated with overall ${ }^{18}$ F-FLUT SUVR (t-tau rho 0.29, $p=0.07$, p-tau rho 0.24, $p=0.15)$.

\section{Hippocampal Volume and Cortical Thickness}

Magnetic resonance imaging data from the two cohorts were analyzed separately due to the use of different scanners for the imaging acquisition. There were no significant differences in mean hippocampal volume or thickness in the six cortical ROIs between SCD and MCI in either of the cohorts (Supplementary Table) and none of the ROIs were significant predictors of the SCD/MCI distinction in binary logistic regression models even after adjustment for age. In Cohort 1 there were no significant correlations between $\mathrm{A} \beta 43 / 42$ CSF levels and the structural MRI measurements. In Cohort 2 there were unadjusted moderate positive correlations for both CSF A $\beta 43$ and $A \beta 42$ with total hippocampal volume, thickness of the middle temporal, inferior parietal and inferior frontal cortices with statistical significance after correction for multiple testing in seven ROIs. CSF A $\beta 43$ correlated significantly also with thickness of the ERC and CSF A $\beta 42$ with posterior cingulate cortical thickness. After adjustment for effects of age, however, only the correlations with hippocampal volume, inferior parietal and inferior frontal cortical thickness were nominally significant (Table 4). In the SCD group CSF A $\beta 43$ correlated significantly with hippocampal volume and CSF $A \beta 42$ with thickness of the 

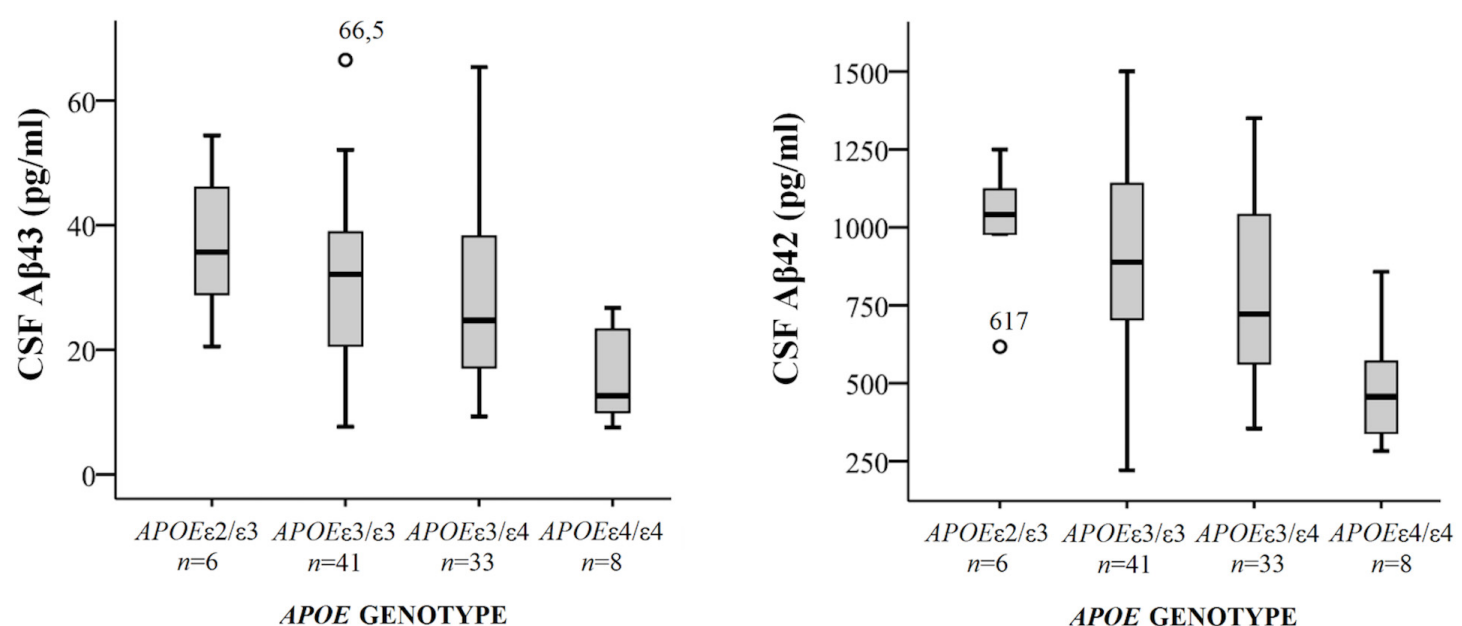

APOE GENOTYPE

FIGURE 1 | Box-plots of cerebrospinal fluid (CSF) A $\beta 43$ and A 342 according to APOE genotype. All subjects from both cohorts have been included, except for one subject with genotype $A P O E \varepsilon 2 / \varepsilon 4$. CSF A 43 for this subject was $17 \mathrm{pg} / \mathrm{ml}$ and CSF A $42598 \mathrm{pg} / \mathrm{ml}$. The whiskers represent the range, except for the two outliers. There were significant differences in mean CSF A 433 and A 342 levels between the $A P O E \varepsilon 4 / \varepsilon 4$-group and the three other groups (all $p<0.01$ ). APOE, Apolipoprotein E genotype.

TABLE 2 | Specification of logistic regression models for the SCD/MCl distinction with one or two CSF biomarkers as covariates together with age.

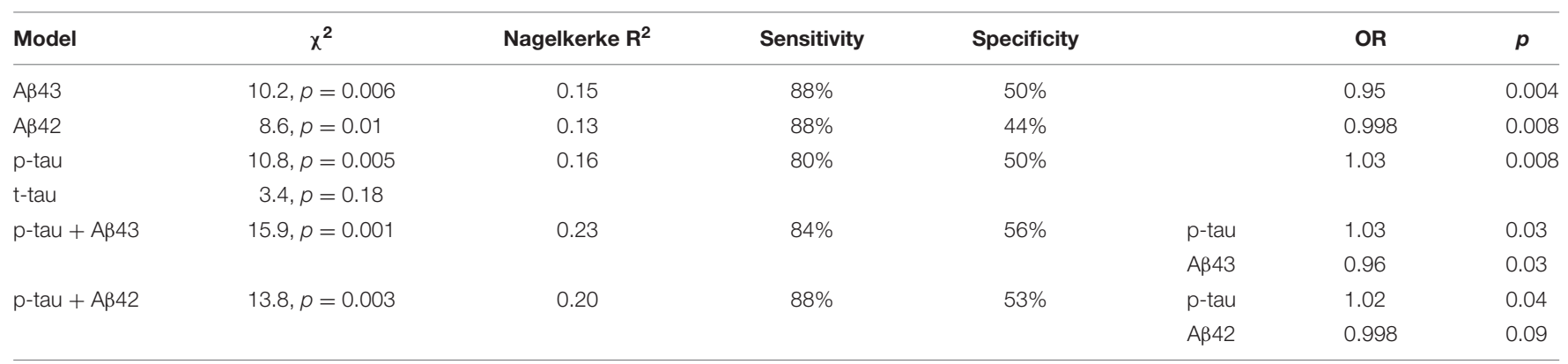

OR, Odds ratio; $\mathrm{MCl}$ coded 1, SCD 0. $n=85$.

TABLE 3 | Correlation analyses between ${ }^{18} \mathrm{~F}$-FLUT SUVRs and A $\beta 43$ or A $\beta 42$ in CSF, unadjusted and adjusted for age.

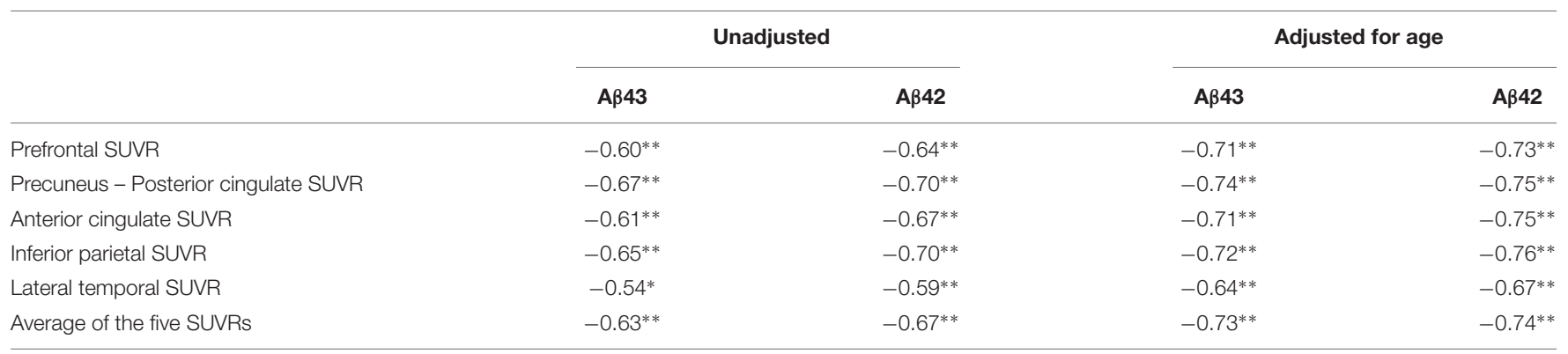

Data presented are Spearman's rank coefficients for correlations between ${ }^{18}$ F-FLUT SUVRs (standardized uptake value ratios) and CSF levels of AB43 and AB42, respectively. Dynamic ${ }^{18}$ F-FLUT data were missing for one subject, $n=39 .{ }^{*}$ Correlations with $p$-values $<0.001 .{ }^{* *}$ Correlations with $p$-values $<0.0001$. There were no statistically significant differences in correlation coefficients between CSF Aß43 and Aß42.

posterior cingulate cortex. In the MCI group there were no significant correlations after correction for age. None of the correlations were significantly different between CSF A 433 and $\mathrm{A} \beta 42$, there was only a trend toward the correlation coefficient for hippocampal volume being stronger with CSF A $\beta 43$ than $\mathrm{A} \beta 42(p=0.05$ unadjusted for age, $p=0.07$ adjusted for age) in the SCD group. Both CSF $A \beta 43$ and $A \beta 42$ were significant predictors of hippocampal volume in linear regression both with and without age in the model. T-tau was a significant predictor when modeled alone and with age, but not when either of the CSF $A \beta$ peptides were entered into the same model. 
TABLE 4 | Correlations between CSF A $\beta 43$, CSF A $\beta 42$, hippocampal volume, and cortical thickness in SCD and MCI subjects together or separately in Cohort 2.

\begin{tabular}{|c|c|c|c|c|c|c|c|}
\hline & & \multicolumn{2}{|c|}{ All } & \multicolumn{2}{|c|}{$\operatorname{SCD} n=11$} & \multicolumn{2}{|c|}{$\mathrm{MCl} n=39$} \\
\hline & & A $\beta 43$ & $A \beta 42$ & A $\beta 43$ & $\mathrm{~A} \beta 42$ & $A \beta 43$ & A $\beta 42$ \\
\hline \multirow[t]{2}{*}{ Hippocampus volume, \%o } & & $0.52^{* *}$ & $0.52^{* *}$ & $0.66^{*}$ & 0.26 & $0.51 * *$ & $0.51^{* *}$ \\
\hline & Age-adjusted & $0.33^{*}$ & $0.30^{*}$ & $0.64^{*}$ & 0.29 & 0.28 & 0.25 \\
\hline \multirow[t]{2}{*}{ Entorhinal cortex thickness } & & $0.39 * *$ & $0.29 *$ & 0.29 & -0.05 & $0.35^{*}$ & 0.23 \\
\hline & Age-adjusted & 0.25 & 0.10 & 0.20 & -0.09 & 0.23 & 0.07 \\
\hline \multirow[t]{2}{*}{ Posterior cingulate cortex thickness } & & $0.37^{*}$ & $0.39 * *$ & 0.47 & $0.66^{*}$ & 0.23 & 0.28 \\
\hline & Age-adjusted & 0.22 & 0.23 & 0.43 & $0.72^{*}$ & 0.02 & 0.04 \\
\hline \multirow[t]{2}{*}{ Temporopolar cortex thickness } & & 0.20 & 0.27 & 0.29 & 0.17 & 0.13 & 0.24 \\
\hline & Age-adjusted & 0.05 & 0.12 & 0.24 & 0.17 & -0.07 & 0.04 \\
\hline \multirow[t]{2}{*}{ Middle temporal cortex thickness } & & $0.46^{* *}$ & $0.51^{* *}$ & 0.52 & 0.34 & $0.43^{* *}$ & $0.51^{* *}$ \\
\hline & Age-adjusted & 0.27 & $0.32^{*}$ & 0.49 & 0.39 & 0.18 & 0.26 \\
\hline \multirow[t]{2}{*}{ Inferior parietal cortex thickness } & & $0.50^{* *}$ & $0.52^{* *}$ & 0.55 & 0.45 & $0.47^{* *}$ & $0.51^{* *}$ \\
\hline & Age-adjusted & $0.30^{*}$ & $0.29 *$ & 0.54 & 0.56 & 0.22 & 0.24 \\
\hline \multirow[t]{2}{*}{ Inferior frontal cortex thickness } & & $0.41^{* *}$ & $0.41^{* *}$ & 0.43 & 0.34 & $0.39 *$ & $0.40^{*}$ \\
\hline & Age-adjusted & $0.32^{*}$ & $0.31 *$ & 0.42 & 0.34 & 0.25 & 0.24 \\
\hline
\end{tabular}

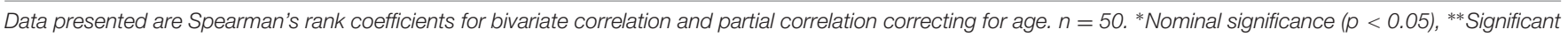

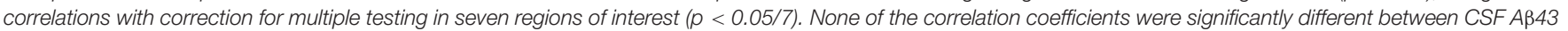
and $A \beta 42$.

\section{Diffusor Tensor Imaging}

Diffusor tensor imaging was only available for Cohort 2. There were no statistically significant differences in the DTI metrics of the selected tracts between the SCD and MCI groups and in logistic regression models none of the DTI metrics were significant covariates for the SCD/MCI distinction even after adjustment for age. Both CSF $A \beta 43$ and $A \beta 42$ were inversely correlated with axial diffusivity in the corticospinal tract and this was the only correlation that maintained significance after correction for multiple testing. Both $A \beta$ peptides showed a nominal significant negative correlation with mean diffusivity in the cingulum bundles and corticospinal tract. There was also a nominal significant negative correlation for CSF A 342 and radial diffusivity, but a positive correlation with fractional anisotropy in the cingulum bundles. Comparing the two $A \beta$ peptides, the positive correlation with fractional

TABLE 5 | Correlations between CSF A $\beta 43$, CSF A $\beta 42$, and diffusion tensor imaging metrics in the selected tracts.

\begin{tabular}{|c|c|c|c|c|c|c|}
\hline & \multicolumn{3}{|c|}{ Unadjusted } & \multicolumn{3}{|c|}{ Adjusted for age } \\
\hline & A $\beta 43$ & $A \beta 42$ & $p$ & A $\beta 43$ & A $\beta 42$ & $p$ \\
\hline FA Corticospinal & 0.11 & 0.17 & - & -0.10 & -0.06 & - \\
\hline FA Callosum-Forceps & 0.21 & $0.30^{*}$ & - & -0.02 & 0.06 & - \\
\hline FA Uncinate fasciculus & -0.02 & 0.01 & - & -0.19 & -0.18 & - \\
\hline DR Callosum-Forceps & $-0.29^{*}$ & $-0.33^{*}$ & - & -0.07 & -0.10 & - \\
\hline DR Uncinate fasciculus & -0.04 & -0.03 & - & 0.18 & 0.23 & - \\
\hline DA Cingulum & $-0.40^{*}$ & $-0.32 *$ & - & -0.27 & 0.16 & - \\
\hline DA Corticospinal & $-0.36^{*}$ & $-0.34^{*}$ & - & $-0.43^{* *}$ & $-0.42^{* *}$ & - \\
\hline DA Callosum-Forceps & $-0.36^{*}$ & $-0.34^{*}$ & - & -0.24 & -0.21 & - \\
\hline MD Callosum-Forceps & $-0.31^{*}$ & $-0.34^{*}$ & - & -0.11 & -0.12 & - \\
\hline MD Uncinate fasciculus & -0.13 & -0.10 & - & 0.10 & 0.17 & - \\
\hline
\end{tabular}

Data presented are Spearman's rank coefficients for bivariate correlation and partial correlation correcting for age, and significant p-values for the test of difference between the correlation coefficients for CSF AB43 and AB42. Diffusion tensor imaging measurements were missing for one subject, $n=49$. FA, fractional anisotropy; DR, radial diffusivity; DA; axial diffusivity, MD; mean diffusivity. *Nominal significance $(p<0.05)$, **Significance with Bonferroni correction for multiple testing ( $p<0.05 / 16)$. 
anisotropy in the cingulum bundles was slightly stronger for CSF A $\beta 42$ compared to CSF $A \beta 43$, though it did not reach significance after strict Bonferroni correction for multiple testing (Table 5).

\section{Cortical Glucose Metabolism}

As the ${ }^{18}$ F-FDG PET scans were obtained on different scanners in the two cohorts, the data were analyzed in each cohort separately. There were no significant differences in overall ${ }^{18} \mathrm{~F}$ FDG SUVR between the SCD and MCI groups and adjustment for age did not change this. There were no significant correlations between ${ }^{18}$ F-FDG SUVRs and either CSF A $\beta 43$ or CSF A $\beta 42$ in Cohort $1(n=28)$. In the larger Cohort $2(n=50)$ both CSF $A \beta 43$ and $A \beta 42$ appeared to be correlated with glucose metabolism in the hippocampus and several of the cortical ROIs, but this changed after correction for the effect of age when only the correlation between CSF A 342 and ${ }^{18}$ F-FDG uptake in the entorhinal cortex was significant after correction for multiple testing. Looking at the SCD and MCI subjects separately, the correlation between CSF A $\beta 43$ and ${ }^{18}$ F-FDG uptake in the posterior cingulate cortex was nominally significant after correction for age, while the only significant correlation after correction for multiple testing was that between CSF $\mathrm{A} \beta 42$ and ${ }^{18} \mathrm{~F}-\mathrm{FDG}$ uptake in the posterior cingulate cortex in the MCI group (Table 6). By direct comparison none of the differences in correlation coefficients between CSF A $\beta 42$ and $A \beta 43$ were statistically significant. Unadjusted there was an inverse relation between $\mathrm{p}$-tau and overall ${ }^{18} \mathrm{~F}$-FDG uptake (average of the seven ROIs), but after correction for age there were no significant correlations with either p-tau or t-tau.

\section{DISCUSSION}

The interest in CSF $\mathrm{A} \beta 43$ as a biomarker first arose from experimental data suggesting that this peptide could be more prone to aggregation than $\mathrm{A} \beta 42$, and thus potentially have importance for amyloidogenesis in AD (Saito et al., 2011; Zou et al., 2013; Conicella and Fawzi, 2014; Burnouf et al., 2015). Our results show that CSF $\mathrm{A} \beta 43$ levels are inversely correlated with cortical amyloid deposits, even at the stage of SCD and before extensive amyloid pathology is evident. However, results revealed nothing to support the hypothesis that the amyloidogenic impact of $A \beta 43$ is different to that of $A \beta 42$. The strength of the correlation between CSF A $\beta 42$ and amyloid load was comparable in the present study to that reported in other studies (Jagust et al., 2009; Tolboom et al., 2009; Landau et al., 2013; Palmquist et al., 2014). Investigating the potential role of CSF A $\beta 43$ in very early AD pathology is difficult. It has been suggested that CSF A $\beta 42$ levels start to drop prior to the increase in amyloid tracer uptake (Fagan et al., 2006; Mattsson et al., 2015), but contradictory results have also been presented (Landau et al., 2013). ${ }^{18}$ F-FLUT only binds $A \beta$ when it has formed extensive $\beta$-sheet formations in insoluble fibrils, and does not bind to the soluble $A \beta$ oligomers that are suggested more likely to be the main neurotoxic culprit in AD (Selkoe and Hardy, 2016). Whether A $\beta 43$ in CSF may have an impact on the quantity and toxicity of oligomers cannot be answered by the current imaging techniques. Recently, the first successful use of a monoclonal antibody-based PET ligand, capable of binding soluble $\mathrm{A} \beta$ protofibrils, was demonstrated in two AD mouse models (Sehlin et al., 2016). Future use of similar radioligands in humans could possibly elucidate the impact on oligomers.

TABLE 6 | Correlations between CSF A $\beta 43$, CSF A $\beta 42$, and ${ }^{18}$ F-FDG SUVRs in Cohort 2.

\begin{tabular}{|c|c|c|c|c|c|c|c|}
\hline & & \multicolumn{2}{|c|}{ All } & \multicolumn{2}{|c|}{$\operatorname{SCD} n=11$} & \multicolumn{2}{|c|}{$\mathrm{MCl} n=39$} \\
\hline & & Aß43 & $A \beta 42$ & A $\beta 43$ & $A \beta 42$ & Aß43 & A $\beta 42$ \\
\hline & Age-adjusted & 0.12 & 0.22 & 0.39 & 0.54 & 0.01 & 0.08 \\
\hline \multirow[t]{2}{*}{ Entorhinal } & & $0.35^{*}$ & $0.47^{* *}$ & 0.26 & 0.60 & $0.34^{*}$ & $0.43^{* *}$ \\
\hline & Age-adjusted & 0.25 & $0.38^{* *}$ & 0.29 & 0.60 & 0.14 & 0.24 \\
\hline \multirow[t]{2}{*}{ Temporopolar } & & 0.28 & $0.36^{*}$ & 0.22 & 0.48 & 0.22 & 0.31 \\
\hline & Age-adjusted & 0.14 & 0.23 & 0.26 & 0.49 & -0.03 & 0.05 \\
\hline \multirow[t]{2}{*}{ Middle temporal } & & $0.37^{*}$ & $0.44^{* *}$ & 0.13 & 0.31 & $0.40^{*}$ & $0.46^{* *}$ \\
\hline & Age-adjusted & 0.16 & 0.24 & 0.16 & 0.31 & 0.10 & 0.14 \\
\hline Inferior parietal & & $0.42^{* *}$ & $0.50^{* *}$ & 0.27 & 0.42 & $0.51^{* *}$ & $0.56^{* *}$ \\
\hline \multirow[t]{2}{*}{ Average of all six cortical ROls } & & $0.41^{* *}$ & $0.51^{* *}$ & 0.07 & 0.35 & $0.48^{* *}$ & $0.55^{* *}$ \\
\hline & Age-adjusted & 0.21 & $0.31^{*}$ & 0.09 & 0.35 & 0.19 & 0.26 \\
\hline
\end{tabular}

Data presented are Spearman's rank correlation coefficients for bivariate correlations and partial correlations controlling for age. ${ }^{*}$ Nominal significance $(p<0.05)$,

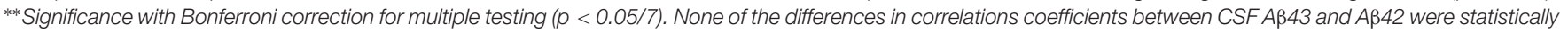
significant. 
Measurements of A $\beta 43$ in CSF have not previously been described in relation to cerebral imaging findings, while CSF $\mathrm{A} \beta 42$ has been extensively studied. CSF $\mathrm{A} \beta 42$ has been shown previously to correlate with hippocampal volume in several cross-sectional studies (Apostolova et al., 2010; Wang et al., 2015). Some longitudinal studies have reported no association between CSF A $\beta 42$ and hippocampal volume at baseline, but an association with subsequent hippocampal atrophy (Schuff et al., 2009; Tosun et al., 2010; Stricker et al., 2012; Mattsson et al., 2014). Other studies have shown no association either at baseline (de Souza et al., 2012) or longitudinally (Henneman et al., 2009; Tarawneh et al., 2015). One explanation for the inconsistency is that the rates of alteration of analytes in CSF and imaging biomarkers are neither parallel nor linear. As a result the correlations between biomarkers will change over time with disease progression (Insel et al., 2016). Divergences in how the various disease stages are defined will further contribute to this variability. We found that the correlation with hippocampal volume tended to be stronger in the SCD group in Cohort 2, especially for CSF A $\beta 43$. Previous studies have shown that brain amyloid load is related to hippocampal volume in cognitively healthy elderly (Dickerson et al., 2009) and in SCD, but not in MCI and AD (Bourgeat et al., 2010; Chételat et al., 2010a). Similarly, Fagan et al. (2009) found that CSF A $\beta 42$ correlated with whole-brain volume in elderly subjects without cognitive impairment, but not in $\mathrm{MCI}$ and $\mathrm{AD}$, suggesting that the association between atrophy and amyloid could be present only early in the disease process. Many studies have described a strong correlation between CSF tau and hippocampal atrophy in MCI and AD (Henneman et al., 2009; Apostolova et al., 2010; de Souza et al., 2012; Tarawneh et al., 2015). In the current study, we found that hippocampal volume was better predicted by either CSF $\mathrm{A} \beta 43$ or $\mathrm{A} \beta 42$ than by $\mathrm{t}$-tau or $\mathrm{p}$-tau, which is in line with former studies in SCD and healthy elderly individuals. That the result differs from past reports in patients with MCI may possibly be attributed to the younger age of our MCI subjects compared to many of the previously published MCI cohorts.

White matter changes are also known to be related to CSF biomarkers (Amlien and Fjell, 2014). CSF A $\beta 42$ has been shown to be positively associated with fractional anisotropy and inversely with mean diffusivity (Gold et al., 2014; Li et al., 2014) as found also in the current study. Both DTI and ${ }^{18}$ F-FLUT PET have been reported to be superior to the core CSF biomarkers in predicting the conversion from MCI to dementia (Selnes et al., 2013; Shaffer et al., 2013; Perani et al., 2016). Therefore, it was particularly interesting to compare these biomarkers with CSF $\mathrm{A} \beta 43$ that in a previous study was suggested to have the same quality. Surprisingly, fractional anisotropy in the cingulum fibers appeared to be better correlated with CSF $A \beta 42$ than $A \beta 43$ and the trend was the same for cortical glucose metabolism.

The current study has several limitations. The use of different MRI and PET-scanners in the two cohorts made direct comparisons between cohorts challenging. Cortical thickness was measured to be higher in Cohort 2 than in Cohort 1 for several of the ROIs even though p-tau levels were on average higher in Cohort 2. Correcting for age resulted in only a slight reduction in the between cohort differences in these
ROIs. It is known that differences in scanner field strength and possibly also scanner settings like pulse sequence, can impact on regional cortical thickness measurements (Han et al., 2006; Govindarajan et al., 2014; McCarthy et al., 2015), which may have contributed to the described differences between the cohorts. The cortical thickness measures were obtained by automated segmentation using the freely available and widely used software FreeSurfer. FreeSurfer version, operating system and workstation used in the processing can also impact the cortical thickness measurements (Gronenschild et al., 2012), but were identical for the two cohorts in this study. Some have suggested that there could be a transitional phase in the development of $\mathrm{AD}$ with increased thickness of certain cortical areas (Chételat et al., 2010b; Fortea et al., 2011; Molinuevo et al., 2012), but this has mainly been described in pre-clinical stages. Because we suspected a significant scanner effect, the imaging data were analyzed in each cohort separately, with a lower number of subjects in each analysis as a consequence. The cohorts came from somewhat different populations; all subjects in Cohort 2 were consecutively recruited from a memory clinic, while Cohort 1 also included subjects recruited by advertisements. Greater variability due to partly community based recruitment and fewer subjects with abnormal CSF biomarkers could be the reason why no correlation with neurodegenerative imaging biomarkers was found in Cohort 1. The study is also limited by the fact that we only included subjects that had already developed cognitive symptoms (which are only subjective in the case of SCD). We could therefore not assess CSF $A \beta 43$ in pre-clinical stages of $A D$ such as in cognitively normal subjects with positive ${ }^{18}$ F-FLUT PET, nor could we evaluate the impact of biomarkers on the distinction between controls and SCD. This ought to be assessed in future studies.

\section{CONCLUSION}

In this first description of CSF $\mathrm{A} \beta 43$ in relation to imaging biomarkers, we found that CSF levels of $A \beta 43$ are inversely correlated with fibrillary $A \beta$ accumulation in the brain and more weakly positively correlated with biomarkers of neurodegeneration including hippocampal volume. However, none of the studied correlations between CSF A $\beta$ and imaging measurements were significantly different between the two $A \beta$ peptides when controlling for multiple testing. We conclude that in respect to imaging, CSF $A \beta 43$ does not appear to contribute any added value over the well-established CSF biomarker A $\beta 42$ in distinguishing individuals with SCD from those with MCI.

\section{AUTHOR CONTRIBUTIONS}

IA planned the study, recruited and clinically examined study participants, performed the statistical analyses and wrote the manuscript. CL planned the study and performed ELISA of CSF A $\beta 43$ together with IM. PS and LK processed the MRI and ${ }^{18} \mathrm{~F}-\mathrm{FDG}$ PET images and performed clinical examinations of participants. CC processed the ${ }^{18} \mathrm{~F}$-FLUT PET images. BG visually interpreted ${ }^{18} \mathrm{~F}-\mathrm{FDG}$ and ${ }^{18} \mathrm{~F}$-FLUT PET scans. MW 
carried out laboratory work and administered the CSF biobank. RG did neuropsychological assessments of participants. AB supervised imaging acquisition. LW, SS, GB, and TF supervised the project. All authors critically revised and approved the manuscript.

\section{FUNDING}

This study is part of a cross-regional collaboration, Dementia Disease Initiation (DDI), and is supported by a grant from the Research Council of Norway (NASATS-NevroNor grant 217780/H10).

\section{REFERENCES}

Albert, M. S., Dekosky, S. T., Dickson, D., Dubois, B., Feldman, H. H., Fox, N. C., et al. (2011). The diagnosis of mild cognitive impairment due to Alzheimer's disease: recommendations from the National Institute on Aging-Alzheimer's Association workgroups on diagnostic guidelines for Alzheimer's disease. Alzheimers Dement. 7, 270-279. doi: 10.1016/j.jalz.2011. 03.008

Amlien, I. K., and Fjell, A. M. (2014). Diffusion tensor imaging of white matter degeneration in Alzheimer's disease and mild cognitive impairment. Neuroscience 276, 206-215. doi: 10.1016/j.neuroscience.2014.02.017

Apostolova, L. G., Hwang, K. S., Andrawis, J. P., Green, A. E., Babakchanian, S., Morra, J. H., et al. (2010). 3D PIB and CSF biomarker associations with hippocampal atrophy in ADNI subjects. Neurobiol. Aging 31, 1284-1303. doi: 10.1016/j.neurobiolaging.2010.05.003

Benton, A. L., and Hamsher, K. (1989). Multilingual Aphasia Examination. Iowa City, IA: AJA Associates.

Blennow, K., Wallin, A., Agren, H., Spenger, C., Siegfried, J., and Vanmechelen, E. (1995). Tau protein in cerebrospinal fluid: a biochemical marker for axonal degeneration?in Alzheimer disease? Mol. Chem. Neuropathol. 26, 231-245.

Bourgeat, P., Chételat, G., Villemagne, V. L., Fripp, J., Raniga, P., Pike, K., et al. (2010). Beta-amyloid burden in the temporal neocortex is related to hippocampal atrophy in elderly subjects without dementia. Neurology 74, 121-127. doi: 10.1212/WNL.0b013e3181c918b5

Bruggink, K. A., Kuiperij, H. B., Claassen, J. A., and Verbeek, M. M. (2013). The diagnostic value of CSF amyloid- $\beta 43$ in differentiation of dementia syndromes. Curr. Alzheimer Res. 10, 1034-1040. doi: 10.2174/15672050113106660168

Burnouf, S., Gorsky, M. K., Dols, J., Grönke, S., and Partridge, L. (2015). A $\beta 43$ is neurotoxic and primes aggregation of A $\beta 40$ in vivo. Acta Neuropathol. 130, 35-47. doi: 10.1007/s00401-015-1419-y

Chemuru, S., Kodali, R., and Wetzel, R. (2016). C-Terminal threonine reduces A $\beta 43$ amyloidogenicity compared with A $\beta 42$. J. Mol. Biol. 428, 274-291. doi: 10.1016/j.jmb.2015.06.008

Chételat, G., Villemagne, V. L., Bourgeat, P., Pike, K. E., Jones, G., Ames, D., et al. (2010a). Relationship between atrophy and beta-amyloid deposition in Alzheimer disease. Ann. Neurol. 67, 317-324. doi: 10.1002/ana.21955

Chételat, G., Villemagne, V. L., Pike, K. E., Baron, J. C., Bourgeat, P., Jones, G., et al. (2010b). Larger temporal volume in elderly with high versus low beta-amyloid deposition. Brain 133, 3349-3358. doi: 10.1093/brain/awq187

Coello, C., Willoch, F., Selnes, P., Gjerstad, L., Fladby, T., and Skretting, A. (2013). Correction of partial volume effect in (18)F-FDG PET brain studies using coregistered MR volumes: voxel based analysis of tracer uptake in the white matter. Neuroimage 72, 183-192. doi: 10.1016/j.neuroimage.2013. 01.043

Conicella, A. E., and Fawzi, N. L. (2014). The C-terminal threonine of A $\beta 43$ nucleates toxic aggregation via structural and dynamical changes in monomers and protofibrils. Biochemistry 53, 3095-3105. doi: 10.1021/bi500131a

Desikan, R. S., Ségonne, F., Fischl, B., Quinn, B. T., Dickerson, B. C., Blacker, D., et al. (2006). An automated labeling system for subdividing the human cerebral cortex on MRI scans into gyral based regions of interest. Neuroimage 31, 968-980.

\section{ACKNOWLEDGMENTS}

The authors thank all the participants in the DDI and MCI projects at Akershus University Hospital for their invaluable contribution and study nurse Erna Utnes for her effort in collecting study data and caring for the participants.

\section{SUPPLEMENTARY MATERIAL}

The Supplementary Material for this article can be found online at: http://journal.frontiersin.org/article/10.3389/fnagi. 2017.00009/full\#supplementary-material

de Souza, L. C., Chupin, M., Lamari, F., Jardel, C., Leclercq, D., Colliot, O., et al. (2012). CSF tau markers are correlated with hippocampal volume in Alzheimer's disease. Neurobiol. Aging 33, 1253-1257. doi: 10.1016/j. neurobiolaging.2011.02.022

Dickerson, B. C., Bakkour, A., Salat, D. H., Feczko, E., Pacheco, J., Greve, D. N., et al. (2009). The cortical signature of Alzheimer's disease: regionally specific cortical thinning relates to symptom severity in very mild to mild AD dementia and is detectable in asymptomatic amyloid-positive individuals. Cereb. Cortex 19, 497-510. doi: 10.1093/cercor/bhn113

Fagan, A. M., Head, D., Shah, A. R., Marcus, D., Mintun, M., Morris, J. C., et al. (2009). Decreased CSF A $\beta 42$ correlates with brain atrophy in cognitively normal elderly. Ann. Neurol. 65, 176-183. doi: 10.1002/ana.21559

Fagan, A. M., Mintun, M. A., Mach, R. H., Lee, S. Y., Dence, C. S., Shah, A. R., et al. (2006). Inverse relation between in vivo amyloid imaging load and cerebrospinal fluid A $\beta 42$ in humans. Ann. Neurol. 59, 512-519. doi: 10.1002/ ana. 20730

Ferreira, D., Perestelo-Pérez, L., Westman, E., Wahlund, L. O., Sarria, A., and Serrano-Aguilar, P. (2014). Meta-review of CSF core biomarkers in Alzheimer's disease: the state-of-the-art after the new revised diagnostic criteria. Front. Aging Neurosci. 6:47. doi: 10.3389/fnagi.2014.00047

Fillenbaum, G. G., van Belle, G., Morris, J. C., Mohs, R. C., Mirra, S. S., Davis, P. C., et al. (2008). Consortium to Establish a Registry for Alzheimer's Disease (CERAD): the first twenty years. Alzheimers Dement. 4, 96-109. doi: 10.1016/j. jalz.2007.08.005

Fischl, B., Salat, D. H., Busa, E., Albert, M., Dieterich, M., Haselgrove, C., et al. (2002). Whole brain segmentation: automated labeling of neuroanatomical structures in the human brain. Neuron 33, 341-355.

Fischl, B., Stevens, A. A., Rajendran, N., Yeo, B. T., Greve, D. N., Van Leemput, K., et al. (2009). Predicting the location of entorhinal cortex from MRI. Neuroimage 47, 8-17. doi: 10.1016/j.neuroimage.2009.04.033

Fischl, B., van der Kouwe, A., Destrieux, C., Halgren, E., Ségonne, F., Salat, D. H., et al. (2004). Automatically parcellating the human cerebral cortex. Cereb. Cortex 14, 11-22.

Folstein, M. F., Folstein, S. E., and McHugh, P. R. (1975). Mini-mental state. A practical method for grading the cognitive state of patients for the clinician. J. Psychiatr. Res. 12, 189-198. doi: 10.1016/0022-3956(75)90026-6

Fortea, J., Sala-Llonch, R., Bartrés-Faz, D., Lladó, A., Solé-Padullés, C., Bosch, B., et al. (2011). Cognitively preserved subjects with transitional cerebrospinal fluid $\beta$-amyloid 1-42 values have thicker cortex in Alzheimer's disease vulnerable areas. Biol. Psychiatry 70, 183-190. doi: 10.1016/j.biopsych.2011.02.017

Fouquet, M., Desgranges, B., Landeau, B., Duchesnay, E., Mezenge, F., Sayette, V., et al. (2009). Longitudinal brain metabolic changes from amnestic mild cognitive impairment to Alzheimer's disease. Brain 132, 2058-2067. doi: 10. 1093/brain/awp132

Gold, B. T., Zhu, Z., Brown, C. A., Andersen, A. H., LaDu, M. J., Tai, L., et al. (2014). White matter integrity is associated with cerebrospinal fluid markers of Alzheimer's disease in normal adults. Neurobiol. Aging 35, 2263-2271. doi: 10.1016/j.neurobiolaging.2014.04.030

Govindarajan, K. A., Freeman, L., Cai, C., Rahbar, M. H., and Narayana, P. A. (2014). Effect of intrinsic and extrinsic factors on global and regional cortical thickness. PLoS ONE 9:e96429. doi: 10.1371/journal.pone.0096429 
Gronenschild, E. H., Habets, P., Jacobs, H. I., Mengelers, R., Rozendaal, N., van Os, J., et al. (2012). The effects of FreeSurfer version, workstation type, and Macintosh operating system version on anatomical volume and cortical thickness measurements. PLOS ONE 7:e38234. doi: 10.1371/journal. pone.0038234

Han, X., Jovicich, K., Salat, D., van der Kouwe, A., Quinn, B., Czanner, S., et al. (2006). Reliability of MRI-derviced measurements of human cerebral thickness: the effect of field strength, scanner upgrade and manufacturer. Neuroimage 32, 180-194. doi: 10.1016/j.neuroimage.2006.02.051

Henneman, W. J. P., Vrenken, H., Barnes, J., Sluimer, I. C., Verwey, N. A., Blankenstein, M. A., et al. (2009). Baseline CSF p-tau levels independently predict progression of hippocampal atrophy in Alzheimer disease. Neurology 73, 935-940. doi: 10.1212/WNL.0b013e3181b879ac

Insel, P. S., Mattson, N., Mackin, R. S., Schöll, M., Nosheny, R. L., Tosun, D., et al. (2016). Accelerating rates of cognitive decline and imaging markers associated with $\beta$-amyloid pathology. Neurology 86, 1887-1896. doi: 10.1212/ WNL.0000000000002683

Iizuka, T., Shoji, M., Harigaya, Y., Kawarabayashi, T., Watanabe, M., Kanai, M., et al. (1995). Amyloid beta-protein ending at Thr43 is a minor component of some diffuse plaques in the Alzheimer's disease brain, but is not found in cerebrovascular amyloid. Brain Res. 702, 275-278.

Ikonomovic, M. D., Klunk, W. E., Abrahamson, E. E., Mathis, C. A., Price, J. C., Tsopelas, N. D., et al. (2008). Post-mortem correlates of in vivo PiB-PET amyloid imaging in a typical case of Alzheimer's disease. Brain 131, 1630-1645. doi: 10.1093/brain/awn016

Jagust, W. J., Landau, S. M., Shaw, L. M., Trojanowski, J. Q., Koeppe, R. A., Reiman, E. M., et al. (2009). Relationships between biomarkers in aging and dementia. Neurology 73, 1193-1199. doi: 10.1212/WNL.0b013e3181bc010c

Jessen, F., Amariglio, R. E., van Boxtel, M., Breteler, M., Ceccaldi, M., Chételat, G., et al. (2014). A conceptual framework for research on subjective cognitive decline in preclinical Alzheimer's disease. Alzheimers Dement. 10, 844-852. doi: 10.1016/j.jalz.2014.01.001

Kakuda, N., Shoji, M., Arai, H., Furukawa, K., Ikeuchi, T., Akazawa, K., et al. (2012). Altered $\gamma$-secretase activity in mild cognitive impairment and Alzheimer's disease. EMBO Mol. Med. 4, 344-352. doi: 10.1002/emmm.2012 00214

Kalheim, L. F., Bjørnerud, A., Fladby, T., Vegge, K., and Selnes, P. (2016). White matter hyperintensity microstructure in amyloid dysmetabolism. J. Cereb. Blood Flow Metab. doi: 10.1177/0271678X15627465 [Epub ahead of print].

Kandimalla, R. J., Prabhakar, S., Binukumar, B. K., Wani, W. Y., Gupta, N., Sharma, D. R., et al. (2011). Apo-Eع4 allele in conjunction with A $\beta 42$ and tau in CSF: biomarker for Alzheimer's disease. Curr. Alzheimer Res. 8, 187-196. doi: 10.2174/156720511795256071

Kandimalla, R. J., Prabhakar, S., Wani, W. Y., Kaushal, A., Gupta, N., Sharma, D. R., et al. (2013). CSF levels in the prediction of Alzheimer's disease. Biol. Open 2, 1119-1124. doi: 10.1242/bio.20135447

Keller, L., Welander, H., Chiang, H. H., Tjernberg, L. O., Nennesmo, I., Wallin, A. K., et al. (2010). The PSEN1 I143T mutation in a Swedish family with Alzheimer's disease: clinical report and quantification of $\mathrm{A} \beta$ in different brain regions. Eur. J. Hum. Genet. 18, 1202-1208. doi: 10.1038/ejhg.2010.107

Kiernan, R. J., Mueller, J., Langston, J. W., and Van Dyke, C. (1987). The neurobehavioral cognitive status examination: a brief but quantitative approach to cognitive assessment. Ann. Intern. Med. 107, 481-485.

Landau, S. M., Lu, M., Joshi, A. D., Pontecorvo, M., Mintun, M. A., and Trojanowski, J. Q. (2013). Comparing positron emission tomography imaging and cerebrospinal fluid measurements of $\beta$-amyloid. Ann. Neurol. 74, 826-836.

Lauridsen, C., Sando, S. B., Shabnam, A., Møller, I., Berge, G., Grøntvedt, G. R., et al. (2016). Cerebrospinal fluid levels of amyloid beta 1-43 in patients with amnestic mild cognitive impairment or early Alzheimer's disease: a 2-year follow-up study. Front. Aging Neurosci. 8:30. doi: 10.3389/fnagi.2016.00030

Lee, I. A., and Preacher, K. J. (2013). Calculation for the Test of the Difference between Two Dependent Correlations with One Variable in Common [Computer Software]. Available at: http://quantpsy.org

Lee, S. H., Coutu, J. P., Wilkens, P., Yendiki, A., Rosas, H. D., Salat, D. H., et al. (2015). Tract-based analysis of white matter degeneration in Alzheimer's disease. Neuroscience 301, 79-89. doi: 10.1016/j.neuroscience.2015.05.049

Li, Q. X., Villemagne, V. L., Doecke, J. D., Rembach, A., Sarros, S., Varghese, S., et al. (2015). Alzheimer's disease normative cerebrospinal fluid biomarkers validated in PET amyloid- $\beta$ characterized subjects from the Australian Imaging, Biomarkers and Lifestyle (AIBL) study. J. Alzheimers Dis. 48, 175-187. doi: 10.3233/JAD-150247

Li, X., Li, T. Q., Andreasen, N., Wiberg, M. K., Westman, E., and Wahlund, L. O., (2014). The association between biomarkers in cerebrospinal fluid and structural changes in the brain in patients with Alzheimer's disease. J. Intern. Med. 275, 418-427. doi: 10.1111/joim.12164

Mattsson, N., Insel, P. S., Donohue, M., Landau, S., Jagust, W., Shaw, L. M., et al. (2015). Independent information from cerebrospinal fluid amyloid- $\beta$ and florbetapir imaging in Alzheimer's disease. Brain 138, 772-783. doi: 10.1093/ brain/awu367

Mattsson, N., Insel, P., Nosheny, R., Trojanowski, J. Q., Shaw, L. M., Jack, C. R., et al. (2014). Effects of CSF proteins on brain atrophy rates in cognitively healthy older adults. Neurobiol. Aging 35, 614-622. doi: 10.1016/j.neurobiolaging.2013. 08.027

McCarthy, C. S., Ramprashad, A., Thompson, C., Botti, J. A., Coman, I. L., and Kates, W. R. (2015). A comparison of FreeSurfer-generated data with and without manual intervention. Front. Neurosci. 9:379. doi: 10.3389/fnins.2015. 00379

Miravalle, L., Calero, M., Takao, M., Roher, A. E., Ghetti, B., and Vidar, R. (2005). Amino-terminally truncated Abeta peptide species are the main component of cotton wool plaques. Biochemistry 44, 10810-10821.

Molinuevo, J. L., Sánches-Valle, R., Lladó, A., Fortea, J., Bartrés-Faz, D., and Rami, L. (2012). Identifying earlier Alzheimer's disease: insights from the preclinical and prodromal phases. Neurodegener. Dis. 10, 158-160. doi: 10.1159/ 000332806

Morris, J. C. (1997). Clinical dementia rating: a reliable and valid diagnostic and staging measure for dementia of the Alzheimer type. Int. Psychogeriatr. 9(Suppl. 1), 173-176; discussion 177-178.

Nakaya, Y., Yamane, T., Shiraishi, H., Wang, H. Q., Matsubara, E., Sato, T., et al. (2005). Random mutagenesis of presenilin-1 identifies novel mutants exclusively generating long amyloid beta-peptides. J. Biol. Chem. 280, 19070-19077.

Palmquist, S., Zetterberg, H., Blennow, K., Vestberg, S., Andreasson, U., Brooks, D. J., et al. (2014). Accuracy of brain amyloid detection in clinical practice using cerebrospinal fluid $\beta$-amyloid 42: a cross-validation study against amyloid positron emission tomography. JAMA Neurol. 71, 1282-1289. doi: 10.1001/ jamaneurol.2014.1358

Parvathy, S., Davies, P., Haroutunian, V., Purohit, D. P., Davis, K. L., Mohs, R. C., et al. (2001). Correlation between $A \beta x-40-, A \beta x-42-$, and $A \beta x-43$-containing amyloid plaques and cognitive decline. Arch. Neurol. 58, 2025-2031. doi: 10. 1001/archneur.58.12.2025

Perani, D., Cerami, C., Caminiti, S. P., Santangelo, R., Coppi, E., Ferrari, L., et al. (2016). Cross-validation of biomarkers for the early differential diagnosis and prognosis of dementia in a clinical setting. Eur. J. Nucl. Med. Mol. Imaging 43, 499-508. doi: 10.1007/s00259-015-3170-y

Reitan, R. M., and Wolfson, D. (1985). The Halstead-Reitan Neuropsychological Test Battery. Tucson, AZ: Neuropsychology Press.

Royall, D. R., Mahurin, R. K., and Gray, K. F. (1992). Bedside assessment of executive cognitive impairment: the executive interview. J. Am. Geriatr. Soc. 40, 1221-1226.

Sabuncu, M. R., Desikan, R. S., Sepulcre, J., Yeo, B. T., Liu, H., Schmansky, N. J., et al. (2011). The dynamics of cortical and hippocampal atrophy in Alzheimer disease. Arch. Neurol. 68, 1040-1048. doi: 10.1001/archneurol.2011.167

Saito, T., Suemoto, T., Brouwers, N., Sleegers, K., Funamoto, S., Mihira, N., et al. (2011). Potent amyloidogenicity and pathogenicity of A $\beta 43$. Nat. Neurosci. 14, 1023-1032. doi: 10.1038/nn.2858

Sandebring, A., Welander, H., Winblad, B., Gra, C., and Tjernberg, L. O. (2013). The pathogenic A $\beta 43$ is enriched in familial and sporadic Alzheimer disease. PLoS ONE 8:e55847. doi: 10.1371/journal.pone.0055847

Schmidt, M. (1996). Rey Auditory and Verbal Learning Test. A Handbook. Los Angeles, CA: Western Psychological Services.

Schuff, N., Woerner, N., Boreta, L., Kornfield, T., Shaw, L. M., Trojanowski, J. Q., et al. (2009). Alzheimer's Disease Neuroimaging Initiative. MRI of hippocampal volume loss in early Alzheimer's disease in relation to ApoE genotype and biomarkers. Brain 132, 1067-1077. doi: 10.1093/brain/awp007

Sehlin, D., Fang, X. T., Cato, L., Antoni, G., Lannfelt, L., and Syvänen, S. (2016). Antibody-based PET imaging of amyloid beta in mouse models 
of Alzheimer's disease. Nat. Commun. 7:10759. doi: 10.1038/ncomms 10759

Selkoe, D. J., and Hardy, J. (2016). The amyloid hypothesis of Alzheimer's disease at 25 years. EMBO Mol. Med. 8, 595-608. doi: 10.15252/emmm.201606210

Selnes, P., Aarsland, D., Bjørnerud, A., Gjerstad, L., Wallin, A., Hessen, E., et al. (2013). Diffusion tensor imaging surpasses cerebrospinal fluid as predictor of cognitive decline and medial temporal lobe atrophy in subjective cognitive impairment and mild cognitive impairment. J. Alzheimers Dis. 33, 723-736. doi: 10.3233/JAD-2012-121603

Shaffer, J. L., Petrella, J. R., Sheldon, F. C., Choudhury, K. R., Calhoun, V. D., and Coleman, R. E. (2013). Predicting cognitive decline in subjects at risk for Alzheimer disease by using combined cerebrospinal fluid, MR imaging and PET biomarkers. Radiology 266, 583-591. doi: 10.1148/radiol.12120010

Shimojo, M., Sahara, N., Mizoroki, T., Funamoto, S., Morishima-Kawashima, M., Kudo, T., et al. (2008). Enzymatic characteristics of I213T mutant Presenilin$1 / \gamma$-secretase in cell models and knock-in mouse brains. FAD-linked mutation impairs $\gamma$-site cleavage of APP-CTF $\beta$. J. Biol. Chem. 283, 16488-16496. doi: 10.1074/jbc.M801279200

Sjögren, M., Vanderstichele, H., Agren, H., Zachrisson, O., Edsbagge, M., Wikkelsø, C., et al. (2001). Tau and Abeta42 in cerebrospinal fluid from healthy adults 21 93 years of age: establishment of reference values. Clin. Chem. 47, 1776-1781.

Stricker, N. H., Dodge, H. H., Dowling, N. M., Han, S. D., Erosheva, E. A., and Jagust, W. J. (2012). CSF biomarker associations with change in hippocampal volume and precuneus thickness: implications for the Alzheimer's pathological cascade. Brain Imaging Behav. 6, 599-609. doi: 10.1007/s11682-012-9171-6

Takami, M., Nagashima, Y., Sano, Y., Ishihara, S., Morishima-Kawashima, M., Funamoto, S., et al. (2009). $\gamma$-Secretase: successive tripeptide and tetrapeptide release from the transmembrane domain of $\beta$-carboxyl terminal fragment. J. Neurosci. 29, 13042-13052. doi: 10.1523/JNEUROSCI.2362-09.2009

Tarawneh, R., Head, D., Allison, S., Buckles, V., Fagan, A. M., Ladenson, J. H., et al. (2015). Cerebrospinal fluid markers of neurodegeneration and rates of brain atrophy in early Alzheimer disease. JAMA Neurol. 72, 656-665. doi: 10.1001/jamaneurol.2015.0202

Tolboom, N., van der Flier, W. M., Yaqub, M., Boellaard, R., Verwey, N. A., Blankenstein, M. A., et al. (2009). Relationship of cerebrospinal fluid markers to 11C-PiB and 18F-FDDNP binding. J. Nucl. Med. 50, 1464-1470. doi: 10.2967/ jnumed.109.064360

Tosun, D., Schuff, N., Truran-Sacrey, D., Shaw, L. M., Trojanowski, J. Q., Aisen, P., et al. (2010). Relations between brain tissue loss, CSF biomarkers and the ApoE genetic profile: a longitudinal MRI study. Neurobiol. Aging 31, 1340-1354. doi: 10.1016/j.neurobiolaging.2010.04.030

Vandersteen, A., Masman, M. F., De Baets, G., Jonckheere, W., van der Werf, K., Marrink, S. J., et al. (2012). Molecular plasticity regulates oligomerization and cytotoxicity of the multipeptide-length amyloid- $\beta$ peptide pool. J. Biol. Chem. 287, 36732-36743. doi: 10.1074/jbc.M112.394635

Vanderstichele, H., Van Kerschaver, E., Hesse, C., Davidsson, P., Buyse, M. A., Andreasen, N., et al. (2000). Standardization of measurement of betaamyloid(1-42) in cerebrospinal fluid and plasma. Amyloid 7, 245-258.

Vanmechelen, E., Vanderstichele, H., Davidsson, P., Van Kerschaver, E., Van Der Perre, B., Sjögren, M., et al. (2000). Quantification of tau phosphorylated at threonine 181 in human cerebrospinal fluid: a sandwich ELISA with a synthetic phosphopeptide for standardization. Neurosci. Lett. 285, 49-52.

Vemuri, P., and Jack, C. R. (2010). Role of structural MRI in Alzheimer's disease. Alzheimers Res. Ther. 2:23. doi: 10.1186/alzrt47

Vemuri, P., Wiste, H. J., Weigand, S. D., Shaw, L. M., Trojanowski, J. Q., Weiner, M. W., et al. (2009). MRI and CSF biomarkers in normal, MCI and AD subjects: predicting future clinical change. Neurology 73, 294-301. doi: 10.1212/WNL. 0b013e3181af79e5

Vukovich, R., Perneczky, R., Drzezga, A., Förstl, H., Kurz, A., and Riemenschneider, M. (2009). Brain metabolic correlates of cerebrospinal fluid beta-amyloid 42 and tau in Alzheimer's disease. Dement. Geriatr. Cogn. Disord. 27, 474-480. doi: 10.1159/000218080

Wang, L., Benzinger, T. L., Hassenstab, J., Blazey, T., Owen, C., Liu, J., et al. (2015). Spatially distinct atrophy is linked to $\beta$-amyloid and tau in preclinical Alzheimer disease. Neurology 84, 1254-1260. doi: 10.1212/WNL.0000000000 001401

Warrington, E. K., and James, M. (1991). The Visual Object and Space Perception Battery. Bury St. Edmunds: Thames Valley Test Company.

Welander, H., Frånberg, J., Graff, C., Sundström, E., Winblad, B., and Tjernberg, L. O. (2009). A $\beta 43$ is more frequent than $A \beta 42$ in amyloid plaque cores from Alzheimer disease brains. J. Neurochem. 110, 697-706. doi: 10.1111/j.14714159.2009.06170.x

Whitwell, J. L., Shiung, M. M., Przybelski, S. A., Weigand, S. D., Knopman, D. S., Boeve, B. F., et al. (2008). MRI patterns of atrophy associated with progression to AD in amnestic mild cognitive impairment. Neurology 70, 512-520.

Yakushev, I., Muller, M. J., Buchholz, H. G., Lang, U., Rossmann, H., and Hampel, H. (2012). Stage-dependent agreement between cerebrospinal fluid proteins and FDG-PET findings in Alzheimer's disease. Curr. Alzheimer Res. 9, 241-247.

Zou, K., Liu, J., Watanabe, A., Hiraga, S., Liu, S., Tanabe, C., et al. (2013). A $\beta 43$ is the earliest-depositing A $\beta$ species in APP transgenic mouse brain and is converted to A $\beta 41$ by two active domains of ACE. Am. J. Pathol. 182, 2322-2331. doi: 10.1016/j.ajpath.2013.01.053

Conflict of Interest Statement: The authors declare that the research was conducted in the absence of any commercial or financial relationships that could be construed as a potential conflict of interest.

The reviewer IB and handling Editor declared their shared affiliation, and the handling Editor states that the process nevertheless met the standards of a fair and objective review.

Copyright (c) 2017 Almdahl, Lauridsen, Selnes, Kalheim, Coello, Gajdzik, Møller, Wettergreen, Grambaite, Bjørnerud, Bråthen, Sando, White and Fladby. This is an open-access article distributed under the terms of the Creative Commons Attribution License (CC BY). The use, distribution or reproduction in other forums is permitted, provided the original author(s) or licensor are credited and that the original publication in this journal is cited, in accordance with accepted academic practice. No use, distribution or reproduction is permitted which does not comply with these terms. 